\title{
INTRAVENOUS MICAFUNGIIN VERSUS VORICONAZOLE FOR CHRONIC PULMONARY ASPERGILLOSIS: A MULTICENTER TRIAL IN JAPAN
}

Shigeru Kohno, ${ }^{1}$ Koichi Izumikawa, ${ }^{1}$ Kenji Ogawa, ${ }^{2}$ Atsuyuki Kurashima, ${ }^{3}$ Niro

Okimoto, ${ }^{4}$ Ryoichi Amitani, ${ }^{5}$ Hiroshi Kakeya, ${ }^{1}$ Yoshihito Niki ${ }^{6}$ and Yoshitsugu Miyazaki ${ }^{7}$ Japan Chronic Pulmonary Aspergillosis Study Group (JCPASG)

\footnotetext{
${ }^{1}$ Department of Molecular Microbiology and Immunology, Nagasaki University Graduate School of Biomedical Sciences, Nagasaki; ${ }^{2}$ Department of Pulmonary Medicine, National Hospital Organization Higashinagoya National Hospital, Nagoya; ${ }^{3}$ Department of Respiratory Diseases, National Hospital Organization Tokyo National Hospital, Tokyo;

${ }^{4}$ Center of Respiratory Diseases, Kawasaki Hospital, Okayama; ${ }^{5}$ Department of Respiratory Medicine, Osaka Red Cross Hospital, Osaka; ${ }^{6}$ Department of Clinical Infectious Diseases, Showa University and ${ }^{7}$ Department of Chemotherapy and Mycoses, National Institute of Infectious Diseases, Tokyo- all in Japan.
}

\section{RUNNING TITLE}

comparison intravenous MCFG and VRCZ for CPA 
*Corresponding author:

Shigeru KOHNO, M.D.

Department of Molecular Microbiology and Immunology

Nagasaki University Graduate School of Biomedical Sciences

1-7-1 Sakamoto

Nagasaki 852-8501

JAPAN

Phone: +8195 (819) 7273

Fax: +8195 (849) 7285

E-mail: s-kohno@nagasaki-u.ac.jp 


\section{SUMMARY}

Chronic pulmonary aspergillosis (CPA) is slowly progressive inflammatory pulmonary syndrome due to Aspergillus spp. The evidence regarding CPA treatment is limited. We conducted a randomized, multicenter, open-label trial comparing intravenous micafungin (MCFG) of 150-300 mg once daily with intravenous voriconazole (VRCZ) of $6 \mathrm{mg} / \mathrm{kg}$ twice on Day 1 followed by $4 \mathrm{mg} / \mathrm{kg}$ twice daily for the treatment of 107 inpatients with CPA to compare the efficacy and safety of both drugs as initial treatment in Japan. Treatment effectiveness was defined by clinical, mycological, radiological and serological responses 2 weeks after the initial administration and at the end of therapy. The total of 50 and 47 patients were assigned to the MCFG and VRCZ groups, respectively. The difference in efficacy rates between MCFG and VRCZ was not significant, either after 2 weeks $[68.0 \%$ vs. $58.7 \%$; the absolute difference, $9.3 \%$ with a $95 \%$ confidence interval (CI), -9.97 to $28.58, \mathrm{P}=0.344]$ or at the end of therapy $(60.0 \%$ vs. $53.2 \%$; the absolute difference, $6.8 \%$ with a $95 \% \mathrm{CI},-12.92$ to $26.54, \mathrm{P}=0.499)$. In the safety evaluation, fewer adverse events occurred in the MCFG than VRCZ group (26.4\% vs. $61.1 \%, \mathrm{P}=0.0004)$. MCFG was as effective as VRCZ and significantly safer than as an initial treatment of CPA. (UMIN Clinical Trials Registry number, UMIN000001786.)

\section{KEYWORDS}

chronic pulmonary aspergillosis, micafungin, voriconazole 


\section{INTRODUCTION}

Chronic forms of pulmonary aspergillosis (CPA) are characterized as a slowly progressive inflammatory pulmonary syndrome due to Aspergillus spp. ${ }^{1,2}$ Several names of these chronic forms of disease have been proposed: semi-invasive aspergillosis ${ }^{3}$, chronic necrotizing pulmonary aspergillosis (CNPA) $)^{4}$, simple or complex aspergilloma, and chronic cavitary and fibrosing pulmonary aspergillosis (CCPA and CFPA). ${ }^{5}$ As Hope et al. ${ }^{6}$ indicated apparently distinct entities do not exist for this syndrome and these forms usually overlap. The common characteristics of these forms, however, consist of 1) underlying pulmonary disorders, 2) the status of low-grade immunosuppression, and 3) less than severe findings of angioinvasion in histopathology. ${ }^{1,5,7}$

Numerous clinical cases and few retrospective studies have been reported for the treatment of $\mathrm{CPA}^{8-12}$; however, there are no large-scale clinical trials have been conducted. Although the latest Infectious Diseases Society of America (IDSA) guidelines for the treatment of aspergillosis recommend oral azoles for CPA as primary treatment ${ }^{13}$, the utility of antifungal injections for CPA treatment is not known. Intravenous antifungal agents may possess important role as an induction therapy followed by oral antifungal drugs as maintenance therapy for CPA cases. Several kinds of intravenous antifungal agents active to Aspergillus spp. are available. Micafungin (MCFG) is new semisynthetic lipopeptide antifungal drug in the echinocandin family that has been developed in Japan. ${ }^{14}$ Several in vitro and in vivo studies have shown high levels of antifungal activity against Aspergillus spp. ${ }^{15-17}$ Voriconazole (VRCZ), from a newer generation of triazoles, has also potent anti-aspergillus activity in vivo and in vitro. ${ }^{18,19}$

Our study is the first prospective large-scale trial comparing intravenous MCFG and intravenous VRCZ as induction therapy in CPA patients who require immediate treatment. 


\section{METHODS}

\section{Patients}

From April 1, 2006, to November 30, 2008, we enrolled patients with CPA at 24 Japanese hospitals in this study, which was approved by the ethics committee at each hospital. Every patient provided written informed consent. Astellas, Co. Ltd. (Tokyo, Japan) supported the study with a grant; Astellas, Co. Ltd. was not involved in the design of study, the enrollment of patients, the collection, analysis, interpretation of the data or preparation of the manuscript. All authors vouch for the completeness and accuracy of the data presented.

Patients were eligible for enrollment if they were at least 20 years old and had been given a diagnosis of CPA. The diagnostic criteria of Hope et al. for CPA including chronic CNPA, CCPA, CFPA and complex aspergilloma were used in this study. ${ }^{6}$ Proposed enrollment criteria for prospective clinical studies of CPA by Denning with minor modifications were used for this trial. ${ }^{5}$ Patients with CPA had to fulfill the following conditions: (1) the existence of at least one of the symptoms in the complex consisting of fever, weight loss, sputum, cough, hemoptysis, fatigue, and shortness of breath; (2) new infiltrates, cavity formation, or expansion of pre-existing cavities with or without peri-cavitary infiltrates and adjacent pleural thickening; (3) at least one positive result of serologic tests including the Platelia Aspergillus test (Fujirebio, Tokyo, Japan) for detecting Aspergillus galactomannan antigen by enzyme-linked immunosorbent assay (cut-off value, 1.0), the Aspergillus immunodiffusion system (Microgen Bioproducts, Ltd., Camberley, United Kingdom) for anti-Aspergillus antibody detection, and the (1,3)- $\beta$-D-glucan test (cut-off value, $11.0 \mathrm{pg} / \mathrm{ml}$; Wako Pure Chemical Industries, Osaka, Japan ; and cut-off value, $20 \mathrm{pg} / \mathrm{ml}$ for the Fungitec G Test, Seikagaku Corporation, Tokyo, 
Japan) and/or any positive evidence of the existence of Aspergillus spp. by molecular diagnosis, culture, or pathological findings; (4) positive findings of at least one of the inflammation markers such as white blood cell (WBC) counts, value of C-reactive protein (CRP), and erythrocyte sedimentation rate (ESR); (5) lack of improvement of symptoms or signs after at least 3 days administration of broad-spectrum antibiotics. The following patients were excluded from the study: (1) patients who received MCFG or VRCZ within one month before the time of enrollment, (2) patients with simple aspergilloma, invasive pulmonary aspergillosis, or allergic bronchopulmonary aspergillosis, (3) patients with infectious diseases other than aspergillosis, (4) pregnant patients, (5) patients who did not provide informed consent, and (6) patients with liver, kidney, or heart failure, which fulfilled the Grade II level defined in the Common Toxicity Criteria grading system of the National Cancer Institute. ${ }^{20}$

\section{Procedures}

The patients were allocated to either the MCFG or VRCZ treatment group. The randomization of the minimization method was performed at a centralized web site by attending physicians after obtaining the informed consent from each patient. Random numbers were generated by the computer. Stratification factors of severity and past history of treatment of CPA including aspergilloma were used. Severity was defined as mild, moderate and severe. The factors that were used for determining severity were (1) serum albumin value (less than $2.5 \mathrm{~g} / \mathrm{dl}$ ), (2) $\mathrm{SpO}_{2}$ less than $90 \%$ or $\mathrm{PaO}_{2}$ less than 60 Torr, (3) impaired oral administration of drugs, (4) expansion of newer infiltration shadow on chest X-ray film that was more than half of the lung field, (5) the existence of severe complications such as malignancies, cerebral vascular disorders, hepatic diseases, kidney 
diseases or heart failure, and (6) administration of corticosteroids. Mild severity was defined as the patient possessing none of the above factors. Moderate severity was defined as the patient possessing only one of the factors listed above. Severe severity was defined as the patient possessing at least two of the factors listed above. The randomization schedule was generated by a computer to ensure balanced treatment allocation. Briefly, when the difference of number of allocated patients between the MCFG and VRCZ groups was less than two, the patients were completely randomized. Hence, when the difference was more than three, the patients were allocated to the smaller number therapy group at $90 \%$ probability or the bigger number therapy group at $10 \%$ probability.

VRCZ was given intravenously at $6 \mathrm{mg} / \mathrm{kg}$ every $12 \mathrm{~h}$ for $24 \mathrm{~h}$, and then $4 \mathrm{mg} / \mathrm{kg}$ every $12 \mathrm{~h}$. MCFG was given at 150-300 mg per day. Dose of MCFG was accepted to be decreased due to patient's body weight by the decision of the attending physician. The reason that the dose of MCFG was not fixed in this study was that no data was available about the dose effect of MCFG in the treatment of pulmonary aspergillosis. Patients received treatment for at least 2 weeks with a maximum duration of 4 weeks.

Patients were followed up until 4 weeks after the first administration. Clinical assessments were made daily during treatment. Clinical laboratory tests were run once every week. Radiological and mycological investigations were performed at 2 weeks and at the end of treatment with a maximum 4 weeks. Concurrent treatment with antibiotics was prohibited in this study.

The primary efficacy end point was response to treatment, which was classified as 'success' or 'failure' at the end of administration (at least 2 weeks of administration with a maximum duration of 4 weeks). Each case was carefully reviewed by the investigators. All clinical response criteria were defined as previously reported with slight modifications. ${ }^{8}$ 
Briefly, four groups of factors, consisting of (1) clinical, (2) laboratory, (3) radiological, and (4) mycological, were assessed. The efficacy was evaluated for each of the four groups of factors regardless of whether there was a partial or complete response. (1) Clinical factors were clinical symptoms and signs such as hemosputum, cough, dyspnea and fever which were recorded individually by attending physicians and assessed by a data-review committee. Clinical improvement was defined by reduction or disappearing of these symptoms or signs. (2) Laboratory data such as CRP level, WBC counts, and ESR were independently assessed by a data-review committee. Laboratory data improvement was defined by reduction levels of these inflammatory markers. The transition of titer of anti-Aspergillus antibody was not evaluated. (3) Radiological factors were also evaluated individually by a data-review committee. Briefly, improvement was defined only if there was apparent improvement in the newly appeared lesions on radiological images; no transitional changes in lesions, were not considered improvements (4) Mycological factors were determined by culture or histopathological tests using clinical samples such as sputum, bronchoalveolar lavage fluid, and percutaneous aspiration biopsy samples. Mycological improvement was defined by disappearing of Aspergillus from clinical samples. The members of the data-review committee were not blinded to the treatment assignment on the evaluation of the outcomes or side effects.

A 'success' with respect to clinical response at the end of the treatment was defined as follows: improvement in at least two of the four groups of factors without deterioration in other two groups of factors. A 'failure' was defined as a clinical response that did not match with the 'success' definition.

The secondary efficacy end point was response to treatment, which was classified as 'success' or 'failure' at the end of 2 weeks administration. All four groups of factors were 
used as in the primary endpoint; however, the criterion of "success" was changed as follows: improvement in at least one of the four groups of factors without deterioration in other groups of factors.

Adverse events were recorded from randomization until the last day of treatment. These events were classified according to the Common Toxicity Criteria grading system of the National Cancer Institute. ${ }^{20}$

\section{Statistical analysis}

The primary objective was to show the superiority of each drug in the per-protocol population. Because number of CPA patients, data for the efficacy of antifungals to CPA and budget were limited, the sample size for this clinical trial was set on the basis of the frequency of adverse events of both drugs. On the basis of the rate of adverse events, which averaged $35 \%$ for MCFG and $62 \%$ for $\mathrm{VRCZ}$, a power of 0.80 and the requirement to show the superiority of one drug to the comparator group with a significance of $5.0 \%$ for the final analysis were set. A total sample size of 100 patients (50 assigned MCFG and 50 assigned VRCZ) satisfying the criteria for the per-protocol population was calculated to be necessary.

The intention-to-treat population was defined as patients who underwent randomization. The modified intention-to-treat population was defined as patients who received at least one dose of the study drug they were initially assigned to receive and who had a baseline diagnosis of CPA. The per-protocol population was defined as patients who were confirmed to have a baseline diagnosis of CPA, the availability of an investigator's assessment of over all treatment at the end of therapy, at least 14 doses of one of the study drugs, and no prohibited medication. The population included in the safety analysis 
consisted of all patients who received their initial study drug. The investigator's assessments of overall treatment success at the end of therapy and at the end of the first 2 weeks were analyzed with a two-sided $95 \%$ confidence intervals (CI). Non-inferiority of MCFG could be concluded if the two-sided $95 \%$ CI for the difference in the proportions (MCFG minus VRCZ) had a lower boundary above $-15 \%$. Categorical variables were tested by the Fisher's exact test and were expressed as frequencies and proportions. Continuous variables were tested by the Student's t-test and were expressed as means and ranges. A P-value of $<0.05$ was considered statistically significant. 


\section{RESULTS}

\section{Enrollment and baseline characteristics of the patients}

A total of 107 patients were recruited by 24 centers in Japan during study period. A total of 53 and 54 patients were assigned to the MCFG and VRCZ group, respectively; these patients comprised the intention-to-treat population. Trial profile was presented in Figure 1. All 107 patients were recruited for safety analysis. Four and six patients were excluded from the intention-to-treat population and the modified intention-to-treat population, respectively. One case in the VRCZ-treated group lacked the assessment data at the first 2 weeks of treatment; therefore, this case was not evaluated at the secondary endpoint but was evaluated at the primary endpoint. The demographic characteristics and underlying conditions of the patients in the intention-to-treat population are summarized in Table 1. The MCFG and VRCZ groups were well matched and no significance difference was observed in the intention-to-treat and modified intention-to-treat populations (data not shown).

\section{Base-line characteristics of the infection and the serological findings}

Characteristics of the patients with CPA such as symptoms, signs, and inflammation markers and the results of the serum tests prior to the administration of study drugs in the per-protocol populations are summarized in Table 2. There were no significant differences in the clinical characteristics including symptoms, laboratory findings and the serological test result between the MCFG and VRCZ groups.

The results of the culture test and biopsy of respiratory specimens are shown in Table 3. The positive rate of culture tests remained at only $50 \%$. 


\section{Response}

The average administration time was 23.6 days for the MCFG group and 20.6 days for the VRCZ group without statistical difference $(\mathrm{P}=0.105)$. The average dose of $\mathrm{MCFG}$ was $167.4 \mathrm{mg} /$ day (range, 75 - $300 \mathrm{mg} /$ day) and that for VRCZ was $8 \mathrm{mg} / \mathrm{kg} /$ day (range, 214 $613 \mathrm{mg} /$ day). There were four cases, in which $300 \mathrm{mg} /$ day of MCFG was used in this study by the decision of the attending physicians. There was a single case in which 75 $\mathrm{mg} /$ day of MCFG was used due to low body weight of the patient. No single patient underwent surgical resection of the pulmonary legions in this study during the evaluation therapy.

The outcome at end of treatment in the per-protocol populations was not significantly different between the MCFG and VRCZ group (Table 4). In the intention-to-treat population, success rates were $56.6 \%$ (30 of 53 patients) with MCFG and $46.3 \%$ (25 of 54 patients) with VRCZ (absolute difference, 10.3\%; 95\% CI, -8.63 to 29.25). The response rates of each group of factors, (clinical, laboratory, radiological, and mycological) are shown in Table 4. There was no significant difference in the response rates among all factors between the MCFG and VRCZ groups.

The outcome at the end of the first 2 weeks of treatment was not significantly different between the MCFG and the VRCZ group. In the intention-to-treat population, success rates were $64.2 \%$ (34 of 53 patients) with MCFG and 50.0\% (27 of 54 patients) with VRCZ (absolute difference, 14.2\%; 95\% CI, -4.61 to 32.91). The response rates of the four groups of evaluated factors were not significantly different (Table 4).

Because the lower 95\%confidence limit for the difference between MCFG and VRCZ at both endpoints settings was below zero, MCFG was considered to be neither inferior nor superior to VRCZ. 


\section{Safety}

Table 5 shows the treatment-related adverse events and reasons for discontinuation from the study in patients who received at least one dose of study drug. Significantly fewer adverse effects were observed in the MCFG group than in the VRCZ group. Hepatic events were the most frequent adverse events in both the MCFG and VRCZ groups (8 cases in the MCFG group and 15 cases in the VRCZ group). There were four cases in which VRCZ was discontinued due to severe hepatic events (at least one of the values of glutamic oxaloacetic transamidase, glutamic pyruvic transamidase, alkaline phosphatase, or gamma-glutamyl transpeptidase exceeded 2.5 times the value of the upper normal limit). Various visual events including photophobia, xanthopsia, abnormal vision, defective color vision, vision blurred, and visual disturbance occurred only in the VRCZ group and all visual events were transient and resolved without intervention. All of the adverse effects other than visual events recovered during the treatment or after the end of treatment, except in one case with alkaline phosphatase elevation, which was mild. 


\section{DISCUSSION}

In the current study, we recruited patients who basically satisfied the criteria for the clinical study of CPA proposed by Denning et al. ${ }^{5}$ The inclusion criteria in this study were designed to recruit patients who required immediate treatment. Another consideration was made using broad-spectrum antibiotics prior to registration to minimize registering patients with bacterial infections in addition to aspergillosis.

The latest IDSA guidelines for the treatment of aspergillosis recommend oral VRCZ or ITCZ for CPA as primary treatment ${ }^{13}$, however there is no a large-scale clinical trial data to support recommendation. Although the utility of antifungal injections for CPA treatment is not known, intravenous antifungal agents may be used as an induction therapy followed by oral antifungal drugs as maintenance therapy for CPA cases. Additionally, intravenous antifungal drugs will be needed if the patients are refractory to oral antifungal drugs or in severe status. Our study is the first large-scale clinical trial comparing intravenous MCFG and intravenous VRCZ as induction therapy in CPA patients who require immediate treatment. It may be ideal to use intravenous drugs as long as possible, however longer usage is not reasonable due to its high cost. Our study design was to use intravenous antifungals for several weeks (max 4 weeks) in order to see these antifungals may have power to stabilize or improve clinical status of CPA patients with active inflammatory signs.

The reason that the treatment success rates of both MCFG and VRCZ were decreased at the end of treatment compared to those of the first 2 weeks of administration is that the definition of treatment success was different. The criteria for "success" at the end of treatment were stricter than those after 2 weeks of administration by the protocol. No deterioration and improvement in at least two of the clinical, laboratory, radiological, and 
mycological factors were required for "success" clinical efficacy assessment at the end of therapy. Lack of improvement in two factors was still allowed if the other two factors were improved for the "success" evaluation after 2 weeks of administration. The number of deterioration cases in clinical, radiological, and mycological factors was few at 2 weeks and the end of treatment. However, $20 \%$ to $30 \%$ of all cases showed deterioration in laboratory factors in both the MCFG and VRCZ groups at 2 weeks and the end of the treatment, although there were no statistical differences between the MCFG and VRCZ groups (data not shown). These data indicated that the effective rate at 2 weeks and the end of treatment of both drugs might be increased if the serological factors were not counted for evaluation. Because the clinical aspects of CPA are complicated, establishing a reasonable assessment system of the clinical effectiveness of antifungals with objective factors is also challenging. The limitation of this study is that there was no longer follow-up data after the discontinuation of the intravenous drugs. Many of the patients had received the following oral antifungals such as ITCZ capsules or VRCZ tablets after the trial. The relapse rate is important for understanding an ultimate outcome of the patients with CPA and required in the future study.

The overall effective rate of both drugs however, was only around $60 \%$ with average duration periods about 3 weeks. The response rates of evaluation factors after the first 2 weeks and at the end of treatment indicated that longer treatment will increase the response rate of all factors with almost $10 \%$ of improve rate gained except Aspergillus eradication. There were four cases in which a high dose of MCFG (300 mg/day) was used in this study. Three of four patients were successfully treated and there were no major adverse reactions. However, this data is not showing dose effect of MCFG, the population of patients taking a high dose of MCFG is too small to compare the dose effect of MCFG 
in patients with CPA.

Our results indicated apparently fewer adverse effects in the MCFG than VRCZ group, even though the average administration period was longer by 3 days. The incidence rate of adverse events was still significantly lower in MCFG group even with exclusion of visual events, which are unique to VRCZ. VRCZ is metabolized via the cytochrome P450 (CYP) 2C19 isozyme in liver ${ }^{21}$ and the CYP2C19 mutant type is generally found in $60 \%-70 \%$ of Asian populations including Japanese, ${ }^{22}$ which indicates that these poor metabolizers of VRCZ are likely to have more serious liver dysfunction. Our current data showed significantly more occurrence of liver dysfunction in the VRCZ than MCFG group. Although the MCFG group showed a lower incidence rate of adverse effects, one case with disseminated intravascular coagulation (DIC) was reported as a severe adverse effect. DIC occurred 4 days after the discontinuation of MCFG in this case and the patient recovered. Monitoring blood concentration of VRCZ, not performed in current study, might support further understanding of frequency and severity of adverse effects.

Our study also revealed the important clinical aspects of patients with CPA in Japan. The mean age of the patients with CPA was 70.9 years old and most patients were men with malnutrition and pulmonary complications, especially tuberculosis sequelae. Smith et al. showed that tuberculosis sequelae are also a major risk factor in patients with CPA in United Kingdom ${ }^{23}$. Only the Aspergillus antibody test by the Aspergillus immunodiffusion system showed a reasonably high positive rate with $88.6 \%$ of all patients, which resembled the previous results by Denning et al. ${ }^{2,5}$ The common serum antigen detecting tools for invasive Aspergillus infection are not suitable for CPA diagnosis.

In conclusion, this first large-scale prospective clinical trial comparing intravenous MCFG and VRCZ for patients with CPA indicated that MCFG and VRCZ showed good 
effectiveness without statistical difference, although significantly fewer adverse effects were observed in the MCFG group. 


\section{APPENDIX}

Astellas, Co. Ltd. (Tokyo, Japan) supported the study with a grant; the sponsor was not involved in the design of study, the enrollment of patients, the collection, analysis, interpretation of the data or preparation of the manuscript.

The following is a list of JCPASG investigators: Yasuhiro Yamazaki, National Hospital Organization Dohoku National Hospital, Asahikawa; Kunihiko Yoshimura, Toranomon Hospital, Tokyo; Takashi Ogura, Kanagawa Cardiovascular and Respiratory Center, Yokohama; Masahiro Shirai, National Hospital Organization Tenryu Hospital, Hamamatsu; Tatsuo Kato, National Hospital Organization Nagara Medical Center, Gifu; Hiroyuki Taniguchi, Tosei General Hospital, Seto; Hidenori Ibata, National Hospital Organization Mie Chuo Medical Center, Tsu; Michiaki Mishima, Kyoto University Hospital, Kyoto; Yoshio Taguchi, Tenri Hospital, Tenri; Yoshiro Mochiduki, National Hospital Organization Himeji Medical Center, Himeji; Yuji Sugimoto, Tottori Prefecture Central Hospital, Tottori; Tadashi Ishida, Kawasaki Hospital, Okayama; Hiroshi Miyawaki, Kagawa Prefectural Central Hospital, Takamatsu; Yuichi Fukuda, Kitakyushu City Yahata Hospital, Kitakyushu; Kiyoyasu Fukushima, Japanese Red Cross Nagasaki Genbaku Isahaya Hospital, Isahaya; Yuichi Inoue, Isahaya Health Insurance General Hospital, Isahaya; Sawai Toyomitsu, Sasebo City General Hospital, Sasebo; Jun-ichi Kadota, Oita University Hospital, Oita; Shigeki Nakamura, National Hospital Organization Ureshino Medical Center, Ureshino - all in Japan. 


\section{REFERENCES}

1 Denning DW. Chronic forms of pulmonary aspergillosis. Clin Microbiol Infect 2001; 7 Suppl 2:25-31.

2 Hope WW, Walsh TJ, Denning DW. The invasive and saprophytic syndromes due to Aspergillus spp. Med Mycol 2005; 43 Suppl 1:S207-38.

3 Gefter WB, Weingrad TR, Epstein DM, Ochs RH, Miller WT. "Semi-invasive" pulmonary aspergillosis: a new look at the spectrum of aspergillus infections of the lung. Radiology 1981; 140:313-21.

4 Binder RE, Faling LJ, Pugatch RD, Mahasaen C, Snider GL. Chronic necrotizing pulmonary aspergillosis: a discrete clinical entity. Medicine (Baltimore) 1982; 61:109-24.

5 Denning DW, Riniotis K, Dobrashian R, Sambatakou H. Chronic cavitary and fibrosing pulmonary and pleural aspergillosis: case series, proposed nomenclature change, and review. Clin Infect Dis 2003; 37 Suppl 3:S265-80.

6 Hope WW, Kruhlak MJ, Lyman CA, Petraitiene R, Petraitis V, Francesconi A, et al. Pathogenesis of Aspergillus fumigatus and the kinetics of galactomannan in an in vitro model of early invasive pulmonary aspergillosis: implications for antifungal therapy. J Infect Dis 2007; 195:455-66.

7 Saraceno JL, Phelps DT, Ferro TJ, Futerfas R, Schwartz DB. Chronic necrotizing pulmonary aspergillosis: approach to management. Chest 1997; 112:541-8.

8 Kohno S, Masaoka T, Yamaguchi H, Mori T, Urabe A, Ito A, et al. A multicenter, open-label clinical study of micafungin (FK463) in the treatment of deep-seated mycosis in Japan. Scand J Infect Dis 2004; 36:372-9.

9 Izumikawa K, Ohtsu Y, Kawabata M, Takaya H, Miyamoto A, Sakamoto S, et al. 
Clinical efficacy of micafungin for chronic pulmonary aspergillosis. Med Mycol 2007; 45:273-8.

10 Camuset J, Nunes H, Dombret MC, Bergeron A, Henno P, Philippe B, et al. Treatment of chronic pulmonary aspergillosis by voriconazole in nonimmunocompromised patients. Chest 2007; 131:1435-41.

11 Sambatakou H, Dupont B, Lode H, Denning DW. Voriconazole treatment for subacute invasive and chronic pulmonary aspergillosis. Am J Med 2006; 119:527 e517-24.

12 Jain LR, Denning DW. The efficacy and tolerability of voriconazole in the treatment of chronic cavitary pulmonary aspergillosis. J Infect 2006; 52:e133-7.

13 Walsh TJ, Anaissie EJ, Denning DW, Herbrecht R, Kontoyiannis DP, Marr KA, Morrison VA, Segal BH, Steinbach WJ, Stevens DA, van Burik JA, Wingard JR, Patterson TF. Infectious Diseases Society of America. Treatment of aspergillosis: clinical practice guidelines of the Infectious Diseases Society of America. Clin Infect Dis 2008;46:327-60.

14 Tomishima M, Ohki H, Yamada A, Takasugi H, Maki K, Tawara S, et al. FK463, a novel water-soluble echinocandin lipopeptide: synthesis and antifungal activity. $J$ Antibiot (Tokyo) 1999; 52:674-6.

15 Tawara S, Ikeda F, Maki K, Morishita Y, Otomo K, Teratani N, et al. In vitro activities of a new lipopeptide antifungal agent, FK463, against a variety of clinically important fungi. Antimicrob Agents Chemother 2000; 44:57-62.

16 Ikeda F, Wakai Y, Matsumoto S, Maki K, Watabe E, Tawara S, et al. Efficacy of FK463, a new lipopeptide antifungal agent, in mouse models of disseminated candidiasis and aspergillosis. Antimicrob Agents Chemother 2000; 44:614-8. 
17 Matsumoto S, Wakai Y, Nakai T, Hatano K, Ushitani T, Ikeda F, et al. Efficacy of FK463, a new lipopeptide antifungal agent, in mouse models of pulmonary aspergillosis. Antimicrob Agents Chemother 2000; 44:619-21.

18 Espinel-Ingroff A, Johnson E, Hockey H, Troke P. Activities of voriconazole, itraconazole and amphotericin B in vitro against 590 moulds from 323 patients in the voriconazole Phase III clinical studies. J Antimicrob Chemother 2008; 61:616-20.

19 Espinel-Ingroff A. In vitro fungicidal activities of voriconazole, itraconazole, and amphotericin B against opportunistic moniliaceous and dematiaceous fungi. J Clin Microbiol 2001; 39:954-8.

20 ProgramCTE. Common toxicity criteria manual. Version 3.0. Bethesda, MD: National Cancer Institute, 2003.

21 Johnson LB, Kauffman CA. Voriconazole: a new triazole antifungal agent. Clin Infect Dis 2003; 36:630-7.

22 Kimura M, Ieiri I, Mamiya K, Urae A, Higuchi S. Genetic polymorphism of cytochrome P450s, CYP2C19, and CYP2C9 in a Japanese population. Ther Drug Monit 1998; 20:243-7.

23 Smith NL, Denning DW. Underlying conditions in chronic pulmonary aspergillosis, including simple aspergilloma. Eur Respir J 2010; [Epub ahead of print] 


\section{FIGURE LEGENDS}

Figure 1. Trial profile

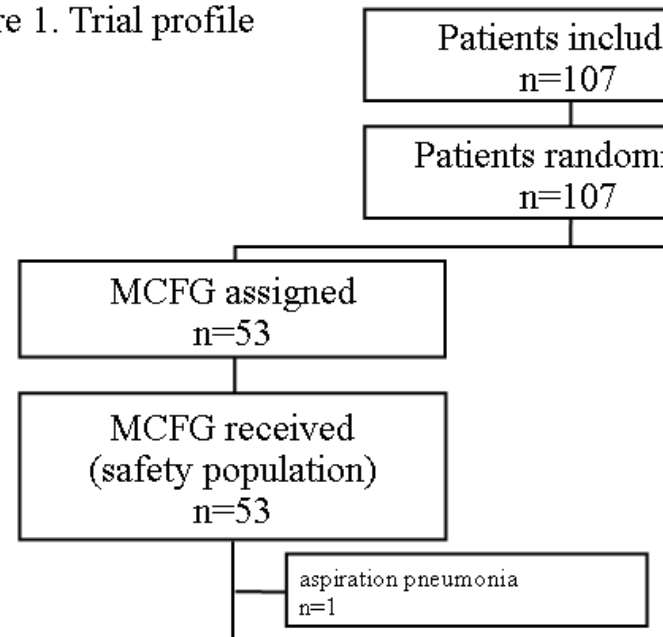

Modified intention-totreat population $n=52$

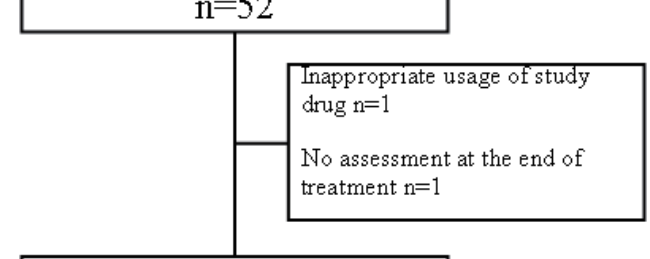

Per-protocol population $\mathrm{n}=50$

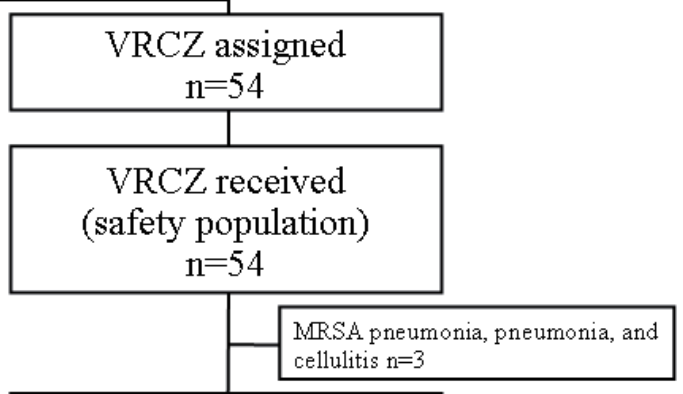

Modified intention-totreat population

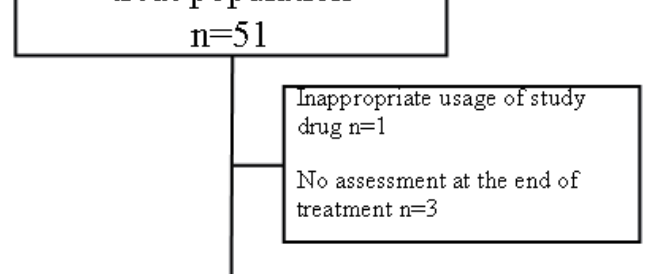

Per-protocol population $\mathrm{n}=47$

MCFG, micafungin; VRCZ, voriconazole; MRSA. methicillin-resistant Staphylococcus aureus 
Table 1. Characteristics of the patients in the intention-to-treat population

\begin{tabular}{|c|c|c|c|c|}
\hline characteristic & overall $(n=107)$ & micafungin $(n=53)$ & voriconazole $(n=54)$ & $\mathrm{P}$ \\
\hline \multicolumn{5}{|l|}{ Age - yr } \\
\hline Mean & 70.9 & 72.1 & 69.9 & 0.163 \\
\hline Range & $47-88$ & $48-87$ & $50-88$ & \\
\hline \multicolumn{5}{|l|}{ Sex - no. $(\%)$} \\
\hline Male & $83(77.6)$ & $37(69.8)$ & $46(85.2)$ & 0.057 \\
\hline Female & $24(22.4)$ & $16(30.2)$ & $8(14.8)$ & \\
\hline \multicolumn{5}{|l|}{ Height - cm } \\
\hline Mean & 159.5 & 159.3 & 159.6 & 0.447 \\
\hline Range & $134.5-179.0$ & $138.0-179.0$ & $134.5-169.5$ & \\
\hline \multicolumn{5}{|l|}{ Weight $-\mathrm{kg}$} \\
\hline Mean & 45.7 & 44.9 & 46.4 & 0.831 \\
\hline Range & $28.0-74.2$ & $28.0-68.0$ & $29.1-74.2$ & \\
\hline \multicolumn{5}{|l|}{ Underlying condition - no. (\%) } \\
\hline Tuberculosis sequelae & $56(52.3)$ & $30(56.6)$ & $26(48.1)$ & 0.441 \\
\hline Chronic obstructive pulmonary disease & $22(20.6)$ & $10(18.9)$ & $12(22.2)$ & 0.812 \\
\hline Diabetes & $13(12.1)$ & $4(7.5)$ & $9(16.7)$ & 0.236 \\
\hline Others & $16(43.0)$ & $22(41.5)$ & $24(44.4)$ & 0.520 \\
\hline
\end{tabular}


Table 2. Clinical characteristics, laboratory and serological findings of the patients in the per-protocol population

\begin{tabular}{|c|c|c|c|c|}
\hline characteristic & overall & micafungin & voriconazole & $\mathrm{P}$ \\
\hline \multicolumn{5}{|l|}{ Body temperature $\left({ }^{\circ} \mathrm{C}\right)$} \\
\hline Number of cases evaluated & 95 & 48 & 47 & \\
\hline Mean & 37.1 & 37.0 & 37.2 & 0.094 \\
\hline Range & $36.2-39.0$ & $36.2-38.2$ & $35.3-39.0$ & \\
\hline \multicolumn{5}{|l|}{ Respiration rate (/ min) } \\
\hline Number of cases evaluated & 61 & 28 & 33 & \\
\hline Mean & 19.7 & 18.8 & 20.5 & 0.181 \\
\hline Range & $12.0-38.0$ & $12.0-28.0$ & $12.0-38.0$ & \\
\hline \multicolumn{5}{|l|}{ Symptoms (\%) } \\
\hline Number of cases evaluated & 97 & 50 & 47 & \\
\hline Cough & $86(88.7)$ & $45(90.0)$ & $41(87.2)$ & \\
\hline Sputum & $82(84.5)$ & $45(90.0)$ & $37(78.3)$ & \\
\hline Hemosputum & $13(13.4)$ & $7(14.0)$ & $6(12.8)$ & \\
\hline Hemoptysis & $0(0)$ & $0(0)$ & $0(0)$ & \\
\hline \multicolumn{5}{|l|}{$\mathrm{SpO}_{2}(\%)$} \\
\hline Number of cases evaluated & 92 & 47 & 45 & \\
\hline Mean & 96.5 & 96.6 & 96.3 & 0.304 \\
\hline Range & $92.0-100.0$ & $92.0-100.0$ & $93.0-99.0$ & \\
\hline \multicolumn{5}{|l|}{ WBC $\left(/ \mathrm{mm}^{3}\right)$} \\
\hline Number of cases evaluated & 97 & 50 & 47 & \\
\hline Mean & 7381.4 & 7051.8 & 7732.1 & 0.308 \\
\hline & $2600-$ & $2600-$ & $3200-$ & \\
\hline Range & 27600 & 14500 & 27600 & \\
\hline \multicolumn{5}{|l|}{ CRP (mg/dl) } \\
\hline Number of cases evaluated & 94 & 49 & 45 & \\
\hline Mean & 4.8 & 4.3 & 5.4 & 0.277 \\
\hline Range & $0.0-21.0$ & $0.0-16.9$ & $0.1-21.0$ & \\
\hline \multicolumn{5}{|l|}{$\operatorname{ESR}(\mathrm{mm} / \mathrm{hr})$} \\
\hline Number of cases evaluated & 59 & 30 & 29 & \\
\hline Mean & 79.8 & 84.0 & 75.4 & 0.408 \\
\hline Range & $7.0-156.9$ & $16.0-138.0$ & $7.0-156.9$ & \\
\hline
\end{tabular}


$\beta$-D-glucan / WAKO (pg/ml)

Number of cases tested

$\begin{array}{cccc}47 & 24 & 23 & \\ 9(19.1 \%) & 7(29.2 \%) & 2(8.7 \%) & 0.137 \\ 12.5 & 17.7 & 7.1 & \\ 0.6-158.3 & 0.6-158.3 & 1.0-33.8 & \end{array}$

$\beta$-D-glucan / Fungitec G test (pg/ml)

Number of cases tested

40

19

21

Positive

$11(27.5 \%)$

$5(26.3 \%)$

$6(28.6 \%) \quad 1.000$

Mean

24.1

22.0

26.0

Range

$0.8-171.0$

$5.0-83.0$

$0.8-171.0$

Platelia Aspergillus test

Number of cases tested

Mean

79

41

38

Range

0.75

0.8

0.7

0.570

$0.1-5.0$

$0.1-5.0$

$0.1-3.9$

Platelia Aspergillus test (\%) cut off: 1.0

Number of cases tested

88

46

42

Positive

$24(27.3 \%) \quad 15(32.6 \%) \quad 9(21.4 \%) \quad 0.338$

Aspergillus immunodiffusion system (\%)

Number of cases tested

79

40

39

Positive

\begin{tabular}{llll}
$70(88.6 \%)$ & $36(90.0 \%)$ & $34(87.2 \%)$ & 0.737 \\
\hline
\end{tabular} 
Table 3. Mycological findings of patients in the per-protocol population overall $(n=84) \quad$ micafungin $(n=45)$ voriconazole $(n=39)$

\section{CULTURE}

A. fumigatus

$30(35.7 \%)$

18

12

A. niger

$4(4.8 \%)$

0

A. terreus

$1(1.2 \%)$

1

Aspergillus spp.

$7(8.3 \%)$

3

22

4

overall Aspergillus spp.

$42(50.0 \%)$

20

\section{BIOPSY}

filamentous fungi

$2(2.4 \%)$

1

1

$\mathrm{n}$ : number of cases in which culture tests were performed on any respiratory specimen. 
Table 4. Treatment success and response rate of clinical, laboratory findings, radiological, and mycological factors at the end of the treatment and two weeks after initial administration in the per-protocol population

\begin{tabular}{lcccc}
\hline & micafungin & voriconazole & Difference in proportion (95\% CI) & P \\
\hline At the end of treatment (primary endpoint) & & & & \\
Number treated successfully (\%) & $30(60.0)$ & $25(53.2)$ & $6.8 \%(-12.92$ to 26.54$)$ & 0.543 \\
Clinical & $35(70.0)$ & $34(72.3)$ & - & 0.826 \\
Laboratory findings & $25(50.0)$ & $25(53.2)$ & - & 0.840 \\
Radiological & $25(50.0)$ & $21(44.7)$ & - & 0.685 \\
Mycological & $9(18.0)$ & $8(17.0)$ & - & 1.000 \\
& & & \\
At the first two weeks (secondary endpoint) & & & - \\
Number treated successfully (\%) & $34(68.0)$ & $27(58.7)$ & $9.3 \%(-9.97$ to 28.58$)$ & 0.399 \\
Clinical & $29(58.0)$ & $27(58.7)$ & - & 1.000 \\
Laboratory findings & $24(48.0)$ & $20(43.5)$ & - & 0.686 \\
Radiological & $20(40.0)$ & $16(34.8)$ & - & 0.675 \\
Mycological & $8(16.0)$ & $4(8.7)$ & & 0.361 \\
\hline
\end{tabular}


Table 5. Treatment-related adverse events and reasons for discontinuation from study of patients who received at least one dose of study drug

\begin{tabular}{|c|c|c|c|c|}
\hline & $\begin{array}{l}\text { micafungin } \\
(\mathrm{n}=53)\end{array}$ & & $\begin{array}{l}\text { voriconazole } \\
\qquad(\mathrm{n}=54)\end{array}$ & $\mathrm{P}$ \\
\hline Serious & $1(1.9 \%)$ & Serious & $4(7.4 \%)$ & \\
\hline \multicolumn{5}{|l|}{ Disseminated intravascular } \\
\hline \multirow[t]{4}{*}{ coagulation } & $1(1.9 \%)$ & Ventricular extrasystoles & $1(1.9 \%)$ & \\
\hline & & Hepatic events & $1(1.9 \%)$ & \\
\hline & & Dizziness & $1(1.9 \%)$ & \\
\hline & & Nausea & $1(1.9 \%)$ & \\
\hline Treatment discontinuation & $3(5.7 \%)$ & Treatment discontinuation & $7(13.0 \%)$ & \\
\hline Rash & $2(3.8 \%)$ & Hepatic events & $4(7.4 \%)$ & \\
\hline \multirow[t]{3}{*}{ Vomiting } & $1(1.9 \%)$ & Hyperkalemia & $1(1.9 \%)$ & \\
\hline & & Ventricular extrasystoles & $1(1.9 \%)$ & \\
\hline & & Dizziness & $1(1.9 \%)$ & \\
\hline Overall & $14(26.4 \%)$ & Overall & $33(61.1 \%)$ & 0.0004 \\
\hline Hepatic events & $8(15.1 \%)$ & Hepatic events & $19(35.2 \%)$ & 0.025 \\
\hline Visual events & $0(0 \%)$ & Visual events & $15(29.6 \%)$ & $<0.0001$ \\
\hline & & Overall excluded visual & & \\
\hline Overall excluded visual events & $14(26.4 \%)$ & events & $27(50 \%)$ & 0.012 \\
\hline
\end{tabular}




$\begin{array}{llll}\text { Rash } & 3(5.7 \%) & \text { Hallucination } & 4(7.4 \%) \\ \text { Vomiting } & 2(3.8 \%) & \text { Dizziness } & 2(3.7 \%) \\ \text { Renal events } & 1(1.9 \%) & \text { Renal events } & 1(1.9 \%) \\ \text { Blood pressure increased } & 1(1.9 \%) & \text { Hyperkalemia } & 1(1.9 \%) \\ \text { Eosinophil count increased } & 1(1.9 \%) & \text { Ventricular extrasystoles } & 1(1.9 \%) \\ \text { White blood cell count decreased } & 1(1.9 \%) & \text { Pseudomembranous colitis } & 1(1.9 \%) \\ \text { Disseminated intravascular } & 1(1.9 \%) & \text { Nausea } & 1(1.9 \%) \\ \text { coagulation } & & \text { Headache } & 1(1.9 \%) \\ & & \text { Restlessness } & 1(1.9 \%) \\ & & \text { Hemoptysis } & 1(1.9 \%) \\ & & \text { Skin discoloration } & 1(1.9 \%) \\ \end{array}$

